\title{
Calcium Burden Assessment and Impact on Drug-Eluting Balloons in Peripheral Arterial Disease
}

\author{
F. Fanelli $\cdot$ A. Cannavale $\cdot$ M. Gazzetti . \\ P. Lucatelli · A. Wlderk $\cdot$ C. Cirelli • \\ A. d'Adamo • F. M. Salvatori
}

Received: 19 February 2014/ Accepted: 2 April 2014/Published online: 9 May 2014

(C) Springer Science+Business Media New York and the Cardiovascular and Interventional Radiological Society of Europe (CIRSE) 2014

\begin{abstract}
Purpose This study was designed to assess the effect of calcium on the efficacy of DEB during revascularization of steno-obstructive SFA lesions.

Methods Sixty patients with de novo lesions of the superficial femoral artery underwent endovascular treatment with drug eluting balloons (DEB). DEB was selected according to vessel reference diameter (1:1). In case of residual stenosis $>50 \%$ or flow-limiting dissection, postdilatation with conventional balloon or provisional stenting was done. Patients were classified into eight groups according to circumferential distribution of calcium
\end{abstract}

F. Fanelli $(\bowtie) \cdot$ A. Cannavale · P. Lucatelli · A. Wlderk ·

C. Cirelli · A. d'Adamo · F. M. Salvatori

Vascular and Interventional Radiology Unit, Department of

Radiologic Sciences, "Sapienza" University of Rome, 324 Viale

Regina Elena, 00161 Rome, Italy

e-mail: fabrizio.fanelli@uniroma1.it

A. Cannavale

e-mail: alessandro.cannavale@ hotmail.com

P. Lucatelli

e-mail: pierleone.lucatelli@gmail.com

A. Wlderk

e-mail: andreawlderk@gmail.com

A. d'Adamo

e-mail: alessandrodadamo@gmail.com

F. M. Salvatori

e-mail: filippomaria.salvatori@uniroma1.it

\section{Gazzetti}

Department of Vascular Surgery, "Sapienza" University of

Rome, 324 Viale Regina Elena, Rome, Italy

e-mail: marianna.gazzetti@uniroma1.it on CT-angiography axial images (from $0^{\circ}$ to $360^{\circ}$ ) and to its length (length $<$ or $>3 \mathrm{~cm}$ ) evaluated with digitalsubtraction-angiography. Ankle-brachial index (ABI), late lumen loss (LLL), target lesion revascularization (TLR), primary (PP) and secondary (SP) patency, major adverse events (MAE), and Rutherford shift were evaluated at 1-year follow-up and correlated with the amount of calcium.

Results Revascularization was successful in all cases. Flow-limiting dissection occurred in five cases $(8.3 \%)$ with a higher circumferential degree of calcium and solved in three cases with postdilatation and in the other two with provisional stenting. DEB effect was lower in patients with higher degree of calcium $\left(>270^{\circ}\right.$ vs. $\left.<90^{\circ}\right)$ : ABI $0.71 \pm 0.07$ versus $0.92 \pm 0.07$; LLL $0.75 \pm 0.21$ versus $0.45 \pm 0.1$; PP 50 versus $100 \%$; SP 50 versus $100 \%$; TLR 25 versus $0 \%$; MAE 25 versus $0 \%$.

Conclusions Calcium represents a barrier to optimal drug absorption. Circumferential distribution seems to be the most influencing factor with the worst effect noticed in $360^{\circ}$ calcium presence.

Keywords Angioplasty · Vascular calcifications · Drug-eluting balloons

\section{Introduction}

Endovascular interventions represent an established practice in the treatment of lower limb peripheral arterial disease (PAD). Several authors confirmed the efficacy of the mini-invasive techniques both in the femoro-popliteal and infrapopliteal regions [1-3].

Among the various contributors of acute and long-term outcomes, calcium represents one of the most widely 
encountered predictors of failure. Despite this, a standard classification of calcium degree is still lacking and calcium is not systematically reported, neither it is always and properly detectable by standard angiography [4].

Still, clear evidence of severe calcification is frequently and unambiguously observed in patients affected by metabolic disorders, such as diabetes and chronic renal disease, and in general in the elderly population. Technical success and patency rates are reported to be lower in case of very calcified arteries [1, 5-7].

While the mechanism of angioplasty (PTA) relies on plaque fracture and vessel wall stretching [4], severe calcium completely alters morphology and compliance of the arterial wall reducing the effect of both angioplasty and stenting. Moreover, it increases the occurrence of flow limiting dissections after PTA [8] and acute vessel recoil, with consequent increased use of stents. Also, in this condition calcium persists in playing an unfavourable role by increasing the risk of stent subexpansion, malposition, and fractures as shown in the coronary environment [5-9].

Many studies have been conducted using different devices to overcome the great difficulty correlated with the presence of calcium. Overall, atherectomy devices, scoring, and cutting balloons have been utilized to improve results and patency rate but without a great success [7-12].

Drug-eluting balloons (DEBs) represent the new generation of very promising devices for the treatment of PAD. Several trials and studies have reported their efficacy to reduce restenosis rate for the treatment of femoro-popliteal and below-the-knee lesions [13-20].

To achieve a durable antiproliferative effect, deep penetration of the drug into the media layers with maximum uptake is required, but calcium may act as a physical barrier to optimal drug penetration and adequate distribution.

The efficacy of DEB in severely calcified arteries has not been specifically assessed with the exception of one registry where the use of DEB after atherectomy was explored in heavily calcific femoro-popliteal arterial segment with the rationale and the initial proof that removal of such a barrier allows drug to penetrate the vessel wall and effectively exert its antiproliferative action [9].

On the basis of this background, we report our experience and results in the evaluation of how DEB performances can be influenced by calcium. Moreover, in the absence of a standardized approach and consensus on calcium assessment and quantification, we propose a calcium scoring method based on CT angiography (CTA) and digital subtraction angiography (DSA) subsequently confirmed by intravascular ultrasound (IVUS) that takes into account longitudinal and circumferential distribution of calcium along the diseased segment.

\section{Materials and Methods}

Study Population

Sixty consecutive patients with de novo steno-obstructive disease (stenosis or occlusion between 3 and $30 \mathrm{~cm}$ in length) of the superficial femoral artery (SFA) were prospectively enrolled in the study. In-stent restenosis, aneurysms, acute thrombosis, pregnancy, life expectancy less than 1 year, and absence of a patent crural artery were the exclusion criteria. Study design was approved by our internal review board.

All patients were informed about the study and the proposed treatment; before the procedure a consent form was signed by each of them.

\section{Study Procedure}

Before the procedure all patients underwent ultrasoundcolor-Doppler (USCD) and CTA to evaluate anatomical characteristics and morphology of the lesions. These characteristics were combined with DSA and IVUS outcomes.

All cases were performed in a state-of-the-art angiosuite using dedicated angiographic equipment (Artis Zee, Siemens AG Medical Solution, Erlangen, Germany). Procedures were done via retrograde common femoral artery access using a 6-Fr/45-cm reinforced introducer sheath (Destination - Terumo, Tokyo, Japan).

After diagnostic angiography and after advancement of the guidewire through the lesion, IVUS assessment with virtual histology (VH-IVUS, Volcano Therapeutics, Rancho Cordova, CA) was done to confirm the morphological characteristics of the vessel. Predilation of the native lesion was performed in all cases using a standard PTA balloon, 1-mm undersized to the reference vessel diameter (RVD).

All procedures were performed using the In.PACT Admiral DEB (Medtronic Invatec, Frauenfeld, Switzerland). Balloon diameter was selected with a 1:1 ratio to RVD. As suggested in other studies and according to the In.PACT study device instructions for use, DEB was kept inflated for at least $60 \mathrm{~s}[15,21]$.

In case of long lesions, requiring more than one DEB, an overlap area of at least $1 \mathrm{~cm}$ was secured between two contiguous balloons. Technical success was confirmed by a residual stenosis $<30 \%$ by visual estimation.

In case of flow-limiting dissection or residual stenosis $>50 \%$, postdilatation or provisional spot stenting was applied. Postdilatation was performed using a conventional, noncoated PTA balloon, with a 1:1 ratio to RVD, which could be repeated to get the final technical success. If postdilatation was not sufficient to achieve the technical 
success, a self-expanding nitinol stent was deployed (Complete SE-Medtronic Inc., Santa Rosa, CA).

\section{Medication}

According to our standard protocol, an intra-arterial bolus of heparin (5,000 IU) was injected immediately after sheath insertion. Aspirin (100 mg/day) and clopidogrel ( $75 \mathrm{mg} /$ day) were administered at least 3 days before the procedure. Patients who were not on an antiplatelet medication had a loading dose of $300 \mathrm{mg}$ of clopidogrel administered just before the procedure.

After treatment, DEB or DEB plus provisional stenting, all patients underwent a medical therapy based on aspirin $(100 \mathrm{mg} /$ day $)$ and clopidogrel $(75 \mathrm{mg} /$ day $)$ for 4 weeks and on aspirin $(100 \mathrm{mg})$ alone thereafter.

Follow-Up

An USCD was done within $24 \mathrm{~h}$ after the procedure to evaluate patency of the vessel, vessel diameter, and possible complications. All patients underwent clinical evaluation and USCD examination at 6 and 12 months followup period.

A CTA was done at 1-year follow-up. In case of abnormal findings at USCD associated with reappearance of symptoms, a DSA was performed.

\section{Study Endpoints}

The primary endpoint was late lumen loss (LLL) at 12 months, evaluated by USCD. LLL was defined as the minimum lumen diameter (MLD) evaluated with USCD within $24 \mathrm{~h}$ after the procedure minus the MLD at 12 months USCD follow-up. MLD was manually measured at USCD in both axial and longitudinal views at the level of the treated lesion.

Secondary endpoints were: 12-month target lesion revascularization (TLR), primary patency (defined as freedom from restenosis by Duplex based on PSVR $<2.4$ and TLR) and secondary patency (defined as freedom from restenosis by Duplex based on PSVR $<2.4$ and TLR for nonocclusive restenosis and irrespective of any revascularization due to occlusion of the target lesion prior to the scheduled 12-month visit), major adverse events (MAE) defined as death, amputation (minor and major), thrombosis, and restenosis rate.

Peak systolic velocity ratio (PSVR) was calculated as the intrastenotic PSV divided by proximally recorded PSV; binary restenosis $(>50 \%)$ was considered when PSVR was equal or higher than 2.4 .
Patients underwent clinical evaluation before and after treatment to analyze the ankle brachial index (ABI) and the Rutherford class shift.

\section{Calcium Burden Evaluation}

To assess the grade of calcium, at the level of the SFA lesion, patients underwent a complete evaluation with CTA, DSA, and IVUS aimed to measure calcium extension both circumferentially and longitudinally.

CTA images, on the axial plane, were used to quantify the circumferential vessel wall distribution of calcium. Two-dimensional axial plane images allow visualization of the different components of the arterial wall, such as calcium, thrombus, and blood flow. A circumferential grade was measured by assessing the presence of calcium in one or more of the four $90^{\circ}$ sectors: grade $1\left(0-90^{\circ}\right)$, grade 2 $\left(0-180^{\circ}\right)$, grade $3\left(0-270^{\circ}\right)$, and grade $4\left(0-360^{\circ}\right.$; Figs. 1 , 2A).

DSA, in the native plain images, was used to evaluate the length of calcium by considering two groups: group A (calcium extending $<3 \mathrm{~cm}$ ) and group $\mathrm{B}$ (calcium extending $>3 \mathrm{~cm}$; Fig. 2B). Figure 3 reports the eight different classes created working out all data according to calcium distribution.

All data acquired with CTA and DSA were confirmed by IVUS that was performed, in all patients, during the interventional procedure using a dedicated catheter $(20 \mathrm{MHz}, 2.9 \mathrm{~F}$ phased-array transducer catheter, Eagle Eye $^{\mathrm{TM}}$ Gold, Volcano Therapeutics, San Diego, CA). Direct visualization of the arterial lumen was done in association with a VH evaluation (Fig. 2C).

\section{Statistical Analysis}

All analyses were performed using SPSS for Windows (SPSS, Chicago, IL) and Excel for Mac 2008. Data analysis was made either per patient, per limb, or per lesion when considered appropriate. No patients were excluded until they reached one of the defined endpoints. Continuous variables were presented as mean with $95 \%$ confidence intervals. Comparison between continuous variables was made by the Student $t$ test. Continuous data are presented as the mean \pm standard deviation (SD) or, if adequate, as the median and the range. For categorical variables, the absolute and relative frequencies were calculated, and comparisons were performed by Fisher's exact test. Comparisons between all groups were analyzed for significant using either parametric analysis of variance or nonparametric Kruskal-Wallis test when appropriate. Significance was assumed at $p<0.05$. 
Fig. 1 Scheme represents the circumferential grade of calcium plaque quantification in four quarters from 0 to 360 degrees
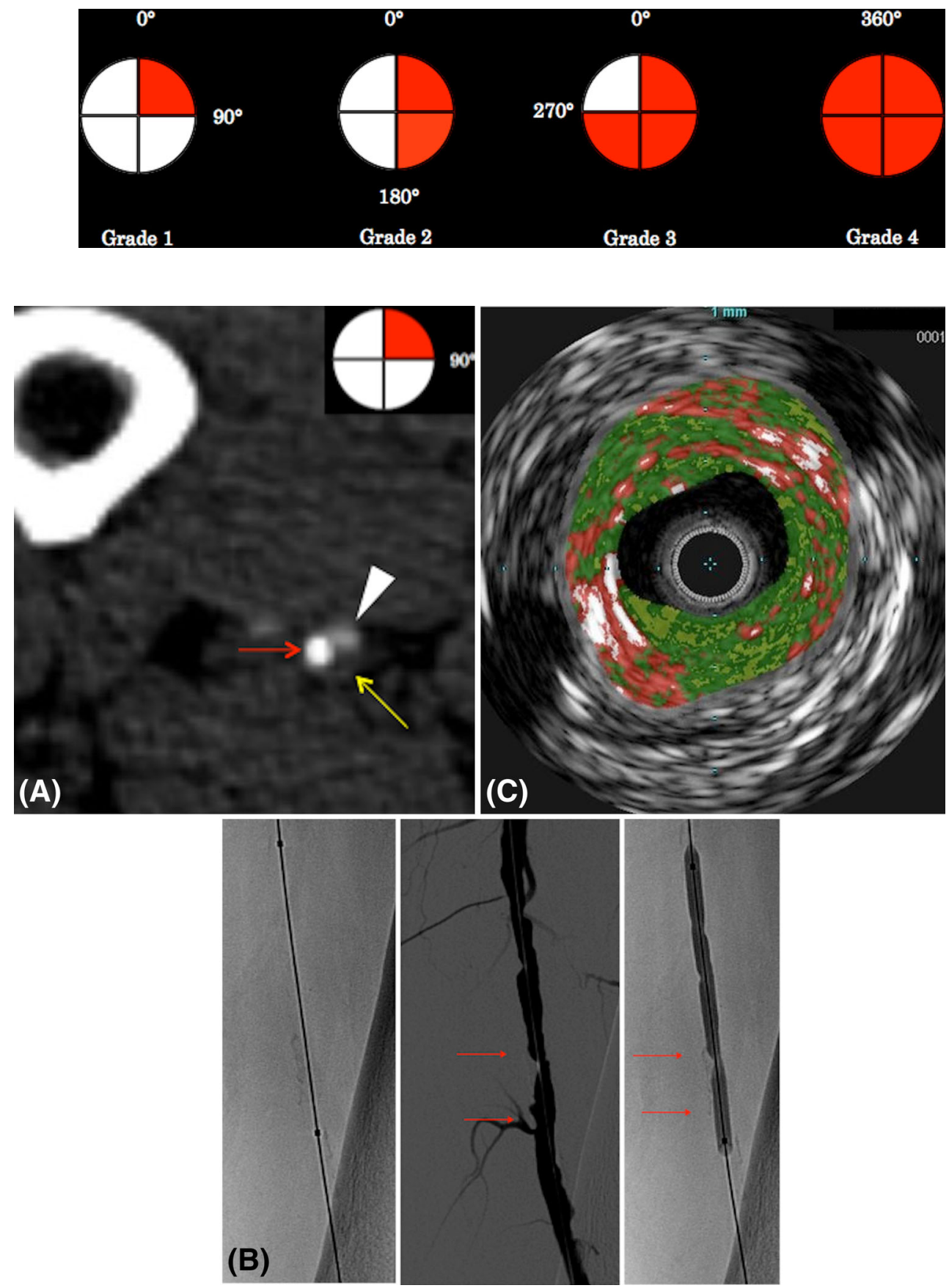

Fig. 2 Sixty-six-year-old male affected by type II diabetes and hypertension under treatment with aspirin, statins, and ACE inhibitor. He recently claimed right limb claudication at $50 \mathrm{~m}$ (ABI of 0.5). A CT angiography revealed a severe stenosis at the mid-distal superficial femoral artery due to a mixed plaque: on axial view the red arrow indicates the calcified part of the plaque, the yellow arrow indicates the soft part and the white arrowhead indicates the residual lumen of the vessel. The small scheme in the right upper corner indicates that calcium burden in this case is $90^{\circ}$. B Stenosis was confirmed by angiography. We also could quantify the length of the whole lesion (arrowheads) and its calcified part $(<3 \mathrm{~cm})$. C IVUS analysis with virtual histology confirmed the angiographic and CT findings thoroughly defining the plaque composition (different histological constituents of the plaque produce different reflected signals and these are assigned different colors defining a colorcoded map: dark green fibrous; yellow/green fibrofatty; white calcified; red necrotic lipid core plaque)

\section{Results}

Sixty patients (44 male), with a mean age of $65 \pm 21$ (range 59-72) years, underwent endovascular treatment with DEB for de novo steno-occlusive disease of the SFA. Several comorbidities (diabetes 51.6\%, high cholesterol $61.6 \%$, hypertension $55 \%$, smoke $41.7 \%$ ) were present in the patients' population.

Patients were divided into eight groups based on the circumferential and longitudinal calcium distribution according to the criteria previously described.
All patients were symptomatic for claudication with a mean baseline $\mathrm{ABI}$ of $0.54 \pm 0.02$. ABI resulted lower, but not significant from a statistical point of view, in those patients with more calcified lesions: $0.55 \pm 0.06$ (group 1a) versus $0.51 \pm 0.03$ (group 4b); $p=0.22$.

In accordance with the Rutherford classification, 4 patients $(6.6 \%)$ were class $2,45(75 \%)$ were class 3 , and $11(18.3 \%)$ were class 4 . Baseline patients' demographic data and clinical characteristics, according to the different groups, are reported in Table 1. 


\begin{tabular}{|c|c|c|}
\hline GROUP & GRADE & LENGTH \\
\hline $1 a$ & \multirow{2}{*}{$0-90^{\circ}$} & $<3 \mathrm{~cm}$ \\
\hline $1 \mathrm{~b}$ & & $>3 \mathrm{~cm}$ \\
\hline 2 a & \multirow{2}{*}{$90-180^{\circ}$} & $<3 \mathrm{~cm}$ \\
\hline $2 \mathrm{~b}$ & & $>3 \mathrm{~cm}$ \\
\hline $3 a$ & \multirow{2}{*}{$180-270^{\circ}$} & $<3 \mathrm{~cm}$ \\
\hline $3 \mathrm{~b}$ & & $>3 \mathrm{~cm}$ \\
\hline 4 a & \multirow{2}{*}{$270-360^{\circ}$} & $<3 \mathrm{~cm}$ \\
\hline $4 \mathrm{~b}$ & & $>3 \mathrm{~cm}$ \\
\hline
\end{tabular}

Fig. 3 Groups classification according to the grade and length of the calcified portion of the lesion

Considering the differences in calcium grade, 5 lesions $(8.3 \%)$ were included in group 1a, $8(13.3 \%)$ in group $1 \mathrm{~b}$, $11(18.3 \%)$ in group $2 \mathrm{a}, 10(16.6 \%)$ in group $2 \mathrm{~b}, 10$ $(16.6 \%)$ in group $3 a, 8(13.3 \%)$ in group $3 b, 4(6.6 \%)$ in group $4 \mathrm{a}$, and $4(6.6 \%)$ in group $4 \mathrm{~b}(p>0.05)$.

Stenoses $(N=41-68.3 \%)$ were more frequent than occlusions $(N=19-31.7 \%)$ and calcium was more evident in stenotic lesions than occlusions. Mean lesion length was $9.0 \pm 4.1 \mathrm{~cm}$; the length of the calcified portion was $6.1 \pm 3.8 \mathrm{~cm}$, ranging from $2.5 \pm 0.5$ to $9.1 \pm 3.9 \mathrm{~cm}$.

In patients with a lesion $<3 \mathrm{~cm}$ (group a) calcified lesion length ranged from $2.5 \pm 0.5$ to $2.8 \pm 0.2 \mathrm{~cm}$, whereas with lesions $>3 \mathrm{~cm}$ (group b) length range was comprised between $4.3 \pm 1.1$ and $9.1 \pm 3.9 \mathrm{~cm}(p<0.05)$.

According to TASC II classification, the majority of the lesions were class B $(N=25-41.6 \%)$ and $\mathrm{C}$ $(N=22-36.6 \%)$. Nine patients $(15.0 \%)$ presented a class A lesion and $4(6.67 \%)$ a class D.

Half of TASC D lesions had calcium extending more than $180^{\circ}$ (group $3 \mathrm{~b}=1$; group $4 \mathrm{~b}=1$ ). Class $\mathrm{A}, \mathrm{B}$, and $\mathrm{C}$ lesions were equally distributed along all the groups (Table 2).

Recanalization of the obstructed segment was done using a subintimal technique in six patients $(10 \%)$. This occurred more frequently in case of longer (group b) calcified lesions (group $1 b=2$; group $2 b=2$; group $3 b=1$; group $4 b=1)$.

After DEB dilatation, in seven patients (11.6\%) a flowlimiting dissection $(N=5-8.3 \%)$ or a residual stenosis $>50 \% \quad(N=2-3.3 \%)$ occurred. Four dissections $(6.6 \%)$ were successfully managed with provisional stenting, whereas in one $(1.6 \%)$ case, postdilatation was sufficient to get technical success. The two residual stenoses were successfully managed with postdilatation. These complications occurred in the most calcified lesions (groups 3 and 4).

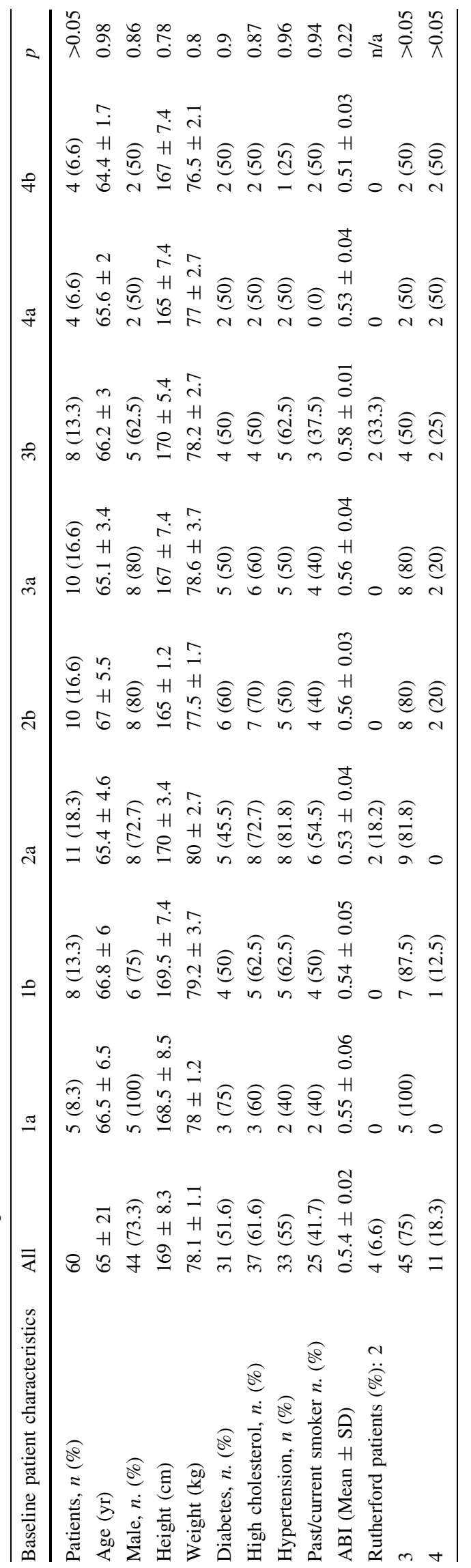


Table 2 Lesions features among all groups

\begin{tabular}{|c|c|c|c|c|c|c|c|c|c|c|}
\hline $\begin{array}{l}\text { Baseline lesion } \\
\text { characteristics }\end{array}$ & All & $1 \mathrm{a}$ & $1 \mathrm{~b}$ & $2 \mathrm{c}$ & $2 b$ & $3 a$ & $3 b$ & $4 a$ & $4 \mathrm{~b}$ & $p$ \\
\hline Lesions, $n .(\%)$ & 60 & $5(8.3)$ & $8(13.3)$ & $11(18.3)$ & $10(16.6)$ & $10(16.6)$ & $8(13.3)$ & $4(6.6)$ & $4(6.6)$ & $>0.05$ \\
\hline $\begin{array}{l}\text { Reference vessel } \\
\text { diameter, mm }\end{array}$ & $5.3 \pm 0.1$ & $5.4 \pm 0.2$ & $5.3 \pm 0.3$ & $5.5 \pm 0.1$ & $5.3 \pm 0.1$ & $5.5 \pm 0.2$ & $5.4 \pm 0.1$ & $5.4 \pm 0.1$ & $5.2 \pm 0.3$ & $<0.01$ \\
\hline Stenosis, $n .(\%)$ & $41(68.3)$ & $1(20)$ & $4(50)$ & $7(63.6)$ & $7(70)$ & $7(70)$ & $7(87.5)$ & $4(100)$ & $4(100)$ & $>0.05$ \\
\hline Occlusions, $n .(\%)$ & $19(31.7)$ & $3(60)$ & $4(50)$. & $5(45.4)$ & $3(30.0)$ & $3(30.0)$ & $1(12.5)$ & 0 & 0 & $>0.05$ \\
\hline $\begin{array}{l}\text { Degree of stenosis, } \\
(\%)\end{array}$ & $87.4 \pm 0.9$ & $86 \pm 0.6$ & $89 \pm 0.9$ & $86 \pm 0.5$ & $87 \pm 0.6$ & $86.5 \pm 1$ & $88 \pm 0.8$ & $87.5 \pm 0.4$ & $88 \pm 0.5$ & $>0.05$ \\
\hline $\begin{array}{l}\text { Lesions length } \\
\text { (calcified), mm } \\
\text { mean } \pm \mathrm{SD}\end{array}$ & $6.1 \pm 3.8$ & $2.6 \pm 0.4$ & $4.3 \pm 1.1$ & $2.8 \pm 0.2$ & $6.2 \pm 3.9$ & $2.6 \pm 0.4$ & $7.3 \pm 2.4$ & $2.5 \pm 0.5$ & $9.1 \pm 3.9$ & $<0.05$ \\
\hline$<7 \mathrm{~cm}$, lesion, $n(\%)$ & $12(20)$ & $5(100)$ & $2(25.0)$ & $8(100)$ & $1(10.0)$ & $10(100)$ & $1(12.5)$ & $4(100)$ & 0 & $>0.05$ \\
\hline $7-15 \mathrm{~cm}$, lesion $n .(\%)$ & $30(50)$ & 0 & $4(50)$ & 0 & $5(50)$ & 0 & $1(12.5)$ & 0 & $2(50)$ & $>0.05$ \\
\hline$>15 \mathrm{~cm}$, lesion $n .(\%)$ & $18(30)$ & 0 & $2(25)$ & 0 & $4(40)$ & 0 & $6(75)$ & 0 & $2(50)$ & $>0.05$ \\
\hline $\begin{array}{l}\text { TASC class* no. of } \\
\text { lesions }(\%) \text { : A }\end{array}$ & $9(15)$ & $3(60)$ & $1(12.5)$ & $2(18.2)$ & 0 & $2(20)$ & 0 & $1(25)$ & 0 & $>0.05$ \\
\hline B & 25 (41.6) & $2(40)$ & $5(62.5)$ & 7 (63.6) & $3(30)$ & $5(50)$ & $2(25)$ & $1(25)$ & 0 & $>0.05$ \\
\hline $\mathrm{C}$ & $22(36.6)$ & 0 & $2(25)$ & $2(18.2)$ & $5(50)$ & $3(30)$ & $5(62.5)$ & $2(50)$ & $3(75)$ & $>0.05$ \\
\hline $\mathrm{D}$ & $4(6.67)$ & 0 & 0 & 0 & $2(10)$ & 0 & $1(12.5)$ & 0 & $1(25)$ & $>0.05$ \\
\hline
\end{tabular}

*TASC II-2007

Table 3 Treatment characteristics and complications according to the different types of lesions

\begin{tabular}{|c|c|c|c|c|c|c|c|c|c|c|}
\hline Treatment characteristics & All & $1 \mathrm{a}$ & $1 b$ & $2 \mathrm{a}$ & $2 b$ & $3 a$ & $3 b$ & $4 a$ & $4 b$ & $p$ \\
\hline PTA & 60 & $5(8.3)$ & $8(13.3)$ & $11(18.3)$ & $10(16.6)$ & $10(16.6)$ & $8\left(\begin{array}{ll}13 & 3\end{array}\right)$ & $4(6.6)$ & $4(6.6)$ & $>0.05$ \\
\hline Subintimal recanalization & $6(10)$ & 0 & $2(25)$ & 0 & $2(10)$ & 0 & $1(12.5)$ & 0 & $1(25)$ & $>0.05$ \\
\hline $\begin{array}{l}\text { No. of balloon per lesion, } \\
\text { mean } \pm \text { SD }\end{array}$ & $1.7 \pm 0.2$ & $1.5 \pm 0.3$ & $2 \pm 0.4$ & $1.4 \pm 0.4$ & $1.7 \pm 0.3$ & $1.5 \pm 0.2$ & $1.7 \pm 0.1$ & $1.8 \pm 0.2$ & $2 \pm 0.3$ & $<0.05$ \\
\hline Dissection $^{\mathrm{a}} \mathrm{n}(\%)$ & $5(8.3)$ & 0 & 0 & 0 & 0 & $2(20)$ & $1(12.5)$ & $1(25)$ & $1(25)$ & $>0.05$ \\
\hline Provisional stenting ${ }^{\mathrm{b}}, n(\%)$ & $4(6.6)$ & 0 & 0 & 0 & 0 & $1(10)$ & $1(12.5)$ & $1(25)$ & $1(25)$ & $>0.05$ \\
\hline Postdilation $^{\mathrm{c}}$ & $3(5)$ & 0 & 0 & $1(9)$ & 0 & $1\left(\begin{array}{ll}10 & 0\end{array}\right)$ & 0 & $1(25)$ & 0 & $>0.05$ \\
\hline
\end{tabular}

${ }^{a}$ Flow-limiting dissection

b Provisional stenting after postdilatation

c Postdilation performed with conventional balloon

Table 3 reports the treatment characteristics according to the different groups of lesions. At 12 months, all patients were alive and underwent the scheduled follow-up visit. As regards the study primary endpoint, mean LLL was $0.61 \pm 0.15 \mathrm{~mm}(p=0.05)$, ranging from $0.45 \pm 0.10 \mathrm{~mm}$ (group1a) to $0.75 \pm 0.21 \mathrm{~mm}$ (group $4 \mathrm{~b}$ ).

The overall primary patency rate was $88.3 \%$ $(p<0.05)$, with an overall secondary patency of $96.7 \%$ $(p>0.05)$. We observed a very low overall TLR rate: $5 \%-3$ cases $(p>0.05)$; again all in the most calcified lesions: groups $3 \mathrm{~b}, 4 \mathrm{a}$, and $4 \mathrm{~b}$.

A strong correlation between LLL, PP and the severity of calcium was clearly evident with the progressive worsening of both endpoints when calcium grade increased (Fig. 4). No cases of thrombosis or amputation were reported.
Aggregate MAE rate, such as thrombosis, amputation, and death, was $5 \%(N=3)$ : not statistically significant $(p>0.05)$ but always correlated with the degree of calcium (group $3 b=1$, group $4 a=1$; group $4 b=1$ ) Table 4.

ABI increased to a mean value of $0.82 \pm 0.09$ $(p<0.001)$ ranging from $0.92 \pm 0.07$ (group 1a) to $0.071 \pm 0.07$ (group 4b). Clinical improvement was again less evident in the higher calcified groups $(p<0.001)$.

A subanalysis comparing the use of DEB in stenoses versus occlusions reported ABI, LLL, and patency rate better for the stenotic lesions (Table 5). On the contrary, DEB showed a good outcome in both conditions, but the higher complexity of an occlusive disease versus a stenotic one must be obviously take in consideration. 
Fig. 4 This chart shows the inverse relationship between the primary patency and late lumen loss (LLL) with calcium groups after 12 months of follow-up

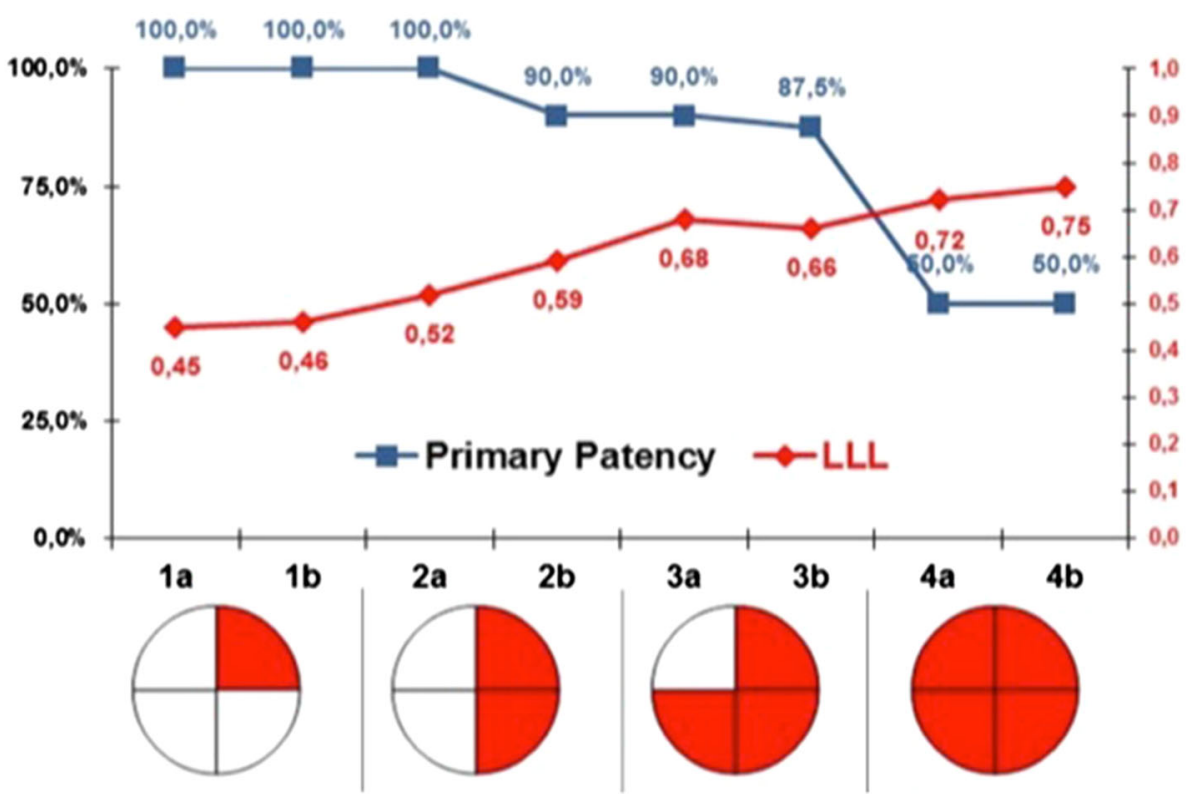

\section{Discussion}

While management of PAD is steadily evolving thanks to the continuous progress in devices and treatment techniques, the high disease burden and the presence of many clinical and anatomical cofactors (such as lesion length, plaque burden, and calcium) continue to represent a challenge and a key element for poor outcomes. Overall, it is calcium that is known for its ability to compromise performances of endovascular procedures in the short and long terms $[8,9]$.

In the past years, very promising results have been reported using DEB in the femoro-popliteal region [19, 20]. Several technical advantages of DEB have been described in their mode of action, such as the ability to achieve a uniform distribution and release of drug on the arterial surface, and to obtain arterial patency with a limited use of stents employed only provisionally [21-23].

A structured and focused assessment of calcium effect on DEB, that is lacking, constitutes the starting point of the present work.

Only few studies have highlighted the importance of calcium $[8,9,15,24]$ and a satisfactory scientific method to measure calcium level has not been found yet. Maybe this is the reason why calcium and its negative influence has not been systematically described and reported in clinical studies.

Rocha-Singh recently described a peripheral arterial calcification scoring systems where intimal and medial vessel wall calcification at the target lesion site is assessed by high intensity fluoroscopy and DSA in the AP projection [24].
However, this scoring system is based only on the length of the calcified portion but do not take in consideration the circumferential distribution. We describe the possibility to classify the amount of calcium in a lesion on the basis of its circumferential and longitudinal distribution.

Recent studies have validated the efficacy of CTA in the evaluation of stenoses in peripheral vessels with high sensitivity and specificity. Also a high accuracy in the assessment of vascular calcium mass and distribution has been highlighted [25, 26]. Using 2D axial images, we were able to quantify the distribution of calcium along the arterial circumference. This is the most important parameter to understand as a completed calcified artery $\left(360^{\circ}\right)$ may compromise DEB efficacy.

The combination with DSA allows the evaluation of the calcium length along the lesion to be completed. We decided to use a cutoff value of $3 \mathrm{~cm}$, because, in our opinion, a calcified portion shorter than $3 \mathrm{~cm}$ can be considered an "easy" lesion, whereas the presence of a longer calcified lesion is more challenging.

According to this method, that in our opinion can be easily reproduced, the eight different groups from $1 \mathrm{a}$ to $4 \mathrm{~b}$ are representative of a progressive 3D increase of calcium, including its circumferential distribution as validated by intralumen IVUS analysis with high specificity and sensitivity [27]. Originally gray-scale IVUS has been considered as the leading endovascular technique for in vivo imaging of the vessel wall in the coronary arteries [28].

However, in the last period, it is going to be applied also for lower limb arterial disease. The availability of more advanced techniques, such as VH software, allows better characterization of the atheromatous plaque. This software 


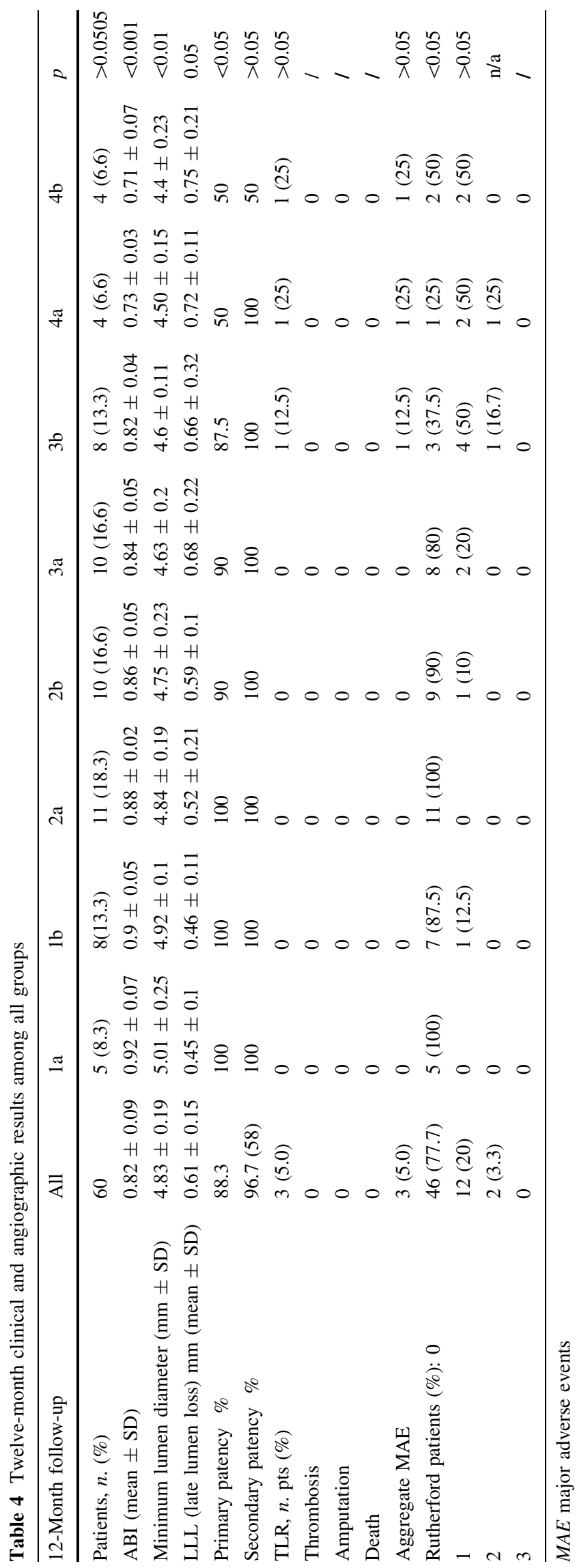

performs in-depth analysis of the backscattered RF-signal in order to provide a more detailed description of the atheromatous plaque composition. It is performed with a 20-MHz, 2.9F phased-array transducer catheter (Eagle Eye $^{\mathrm{TM}}$ Gold, Volcano Therapeutics). These informations are processed determining four basic plaque tissue components with different color codes: (1) fibrous tissue (dark green); (2) fibrofatty tissue (light green); (3) necrotic core (red); and (4) calcium (white). Our analysis shows an inverse correlation between DEB performances and degree of calcium, confirming the efficacy of DEB in reducing LLL but also that calcium lowers the drug activity. In fact, LLL was higher in the most calcified arteries $(p=0.05)$.

This correlation compromises not only the LLL (primary endpoint) and restenosis but also the technical success. In fact, after angioplasty, flow-limiting dissections requiring provisional stenting occurred only in groups 3 and 4, the most calcific lesions. Also, TLR and MAE were observed only in these two groups: groups 3 and 4 .

A deeper analysis confirmed that circumferential distribution of calcium is more important than its length ( $>$ or $<3 \mathrm{~cm}$ ) for DEB efficacy. Flow-limiting dissections were not correlated to the length as they occurred in both a $(<3 \mathrm{~cm})$ and $\mathrm{b}(>3 \mathrm{~cm})$ groups.

In particular, we observed that DEB efficacy can be significantly affected by calcium when it is present on the entire arterial wall $\left(360^{\circ}\right.$; group 4$)$. On the other hand, the presence of a single $90^{\circ}$ calcium-free sector (or an even smaller calcium-free "window") seems enough to allow drug penetration and action as signaled by the marked raise of LLL in group 4 (representing the fully calcium "jailed" arteries) and irrespective of calcium length.

Cioppa et al. reported the results of a single-center experience where DEB was used in combination with directional atherectomy to overcome the difficulties correlated to heavy calcified lesions in the SFA. They concluded that combined treatment is a safe procedure associated with a low restenosis rate [9].

The role of combined therapy represents an interesting approach that mandates further assessments in larger trials to gain a better measure of the incremental value that debulking may carry over DEB alone from both the pure clinical and the cost-effectiveness perspectives.

As much as the correlation between calcium and DEB performance appears clear, overall 12-month LLL of $0.61 \pm 0.15 \mathrm{~mm}$ is similar to the results reported in other studies and obviously better than the LLL reported using conventional angioplasty [13-20].

In recent prospective studies, DEB have shown a primary patency at 1 year comprised between 83.7 [16] and $92.1 \%$ [17]. Other studies comparing DEB versus PTA found a primary patency of DEB significantly higher compared with PTA: Tepe et al. [14] reported a 6-month 
Table 5 Comparative analysis of clinical and angiographic results in stenotic lesions and occlusions

\begin{tabular}{lllll}
\hline 12-Month subanalysis & All & Occlusions & Stenosis & $p$ \\
\hline Lesions, $n,(\%)$ & 60 & $19(31.7)$ & $41(68.3)$ & n/a \\
ABI (mean \pm SD) & $0.82 \pm 0.09$ & $0.79 \pm 0.02$ & $0.85 \pm 0.04$ & $<0.05$ \\
LLL (late lumen loss) mm (mean \pm SD) & $0.61 \pm 0.15$ & $0.71 \pm 0.15$ & $0.5 \pm 0.2$ & $90.2(37)$ \\
Primary patency \% $(n)$ & $88.3(53)$ & $84.2(16)$ & $95.1(2)$ & (2) \\
Secondary patency \% $(n)$ & $96.7(2)$ & 100 & $1(5.9)$ & n/a \\
TLR, $n$. pts $(\%)$ & $3(5)$ & $1(5.3)$ & 2.05 \\
\hline
\end{tabular}

restenosis rate significantly lower in patients treated with DEB than among patients in the control group (17 vs. $44 \%, p=0.01)$. Werk et al. [18] reported a 1-year binary restenosis of $8.6 \%$ for DEB versus $32.4 \%$ for PTA $(p=0.01)$, as well as Fanelli et al. [15], who found in patients with femoropopliteal and infragenicular vascular disease a primary patency rate at 12 months of $76 \%$ for DEB versus $39.6 \%$ for the PTA arm $(p=0.04)$.

In our study, primary patency rate of $88.3 \%$ and secondary patency rate of $96.7 \%$ are noteworthy in consideration of the inclusion in our population of highly calcified lesions. A subanalysis of the primary patency rate, calcium related, reported $100 \%$ for the less calcified lesions (groups 1 and 2) and a mean value of $69.37 \%$ for the heavy calcified ones (groups 3 and 4). Also these data were not correlated with the lesion length.

Stents were rarely used at a rate of $6.6 \%$ overall. However, they were required in $25 \%$ of the heavily calcified subgroup (grade 4). We believe that such low rate of provisional stenting is the result of our renewed and strict dedication to optimal PTA, which we systematically accomplished through slow and long balloon inflation. Moreover when the result was unsatisfactory, postdilation was repeatedly performed (up to 3 times) until the target goal was reached. This technical aspect is extremely important especially in highly calcified lesions.

Our reduced stent rate, while consistent with many previous DEB studies, brings important advantages to patients who unavoidably remain exposed to the likelihood of a future retreatment for the natural progression of PAD disease an event even more relevant when relatively young patients are involved as it was in our series (mean age $65 \pm 21$ years).

\section{Conclusions}

Our study represents the first effort towards a standardized method for a 3D quantification of the calcium grade in SFA lesions. We are able to confirm the unfavorable role of calcium on endovascular procedures in both the short and long terms. Thus, a correlation between calcium degree and DEB performance has been clearly observed, but the efficacy of DEB seems to be particularly compromised only in the presence of a completely $\left(360^{\circ}\right)$ calcified artery. The potential role of a combined therapy, whether with plaque incision or debulking before DEB, warrants further research on both fronts of clinical and cost-effectiveness.

Final overall results in this complicated population with highly calcified lesions remarks the safety and efficacy of DEB in reducing reintervention rate and in improving patency.

Conflict of interest Fabrizio Fanelli, Alessandro Cannavale, Marianna Gazzetti, Pierleone Lucatelli, Andrea Wlderk, Carlo Cirelli, Alessandro d'Adamo, Filippo M. Salvatori have no conflict of interest.

Disclosures The Authors declare that no grant resources have been applied for this manuscript.

\section{References}

1. Schillinger M, Minar E (2012) Percutaneous treatment of peripheral artery disease: novel techniques. Circulation 126(20):2433-2440

2. European Stroke Organisation, Tendera M, Aboyans V, Bartelink ML, Baumgartner I, Clément D, Collet JP, Cremonesi A, De Carlo M, Erbel R, Fowkes FG, Heras M, Kownator S, Minar E, Ostergren J, Poldermans D, Riambau V, Roffi M, Röther J, Sievert H, van Sambeek M, Zeller T (2011) ESC Committee for Practice Guidelines. ESC Guidelines on the diagnosis and treatment of peripheral artery diseases: Document covering atherosclerotic disease of extracranial carotid and vertebral, mesenteric, renal, upper and lower extremity arteries: the Task Force on the Diagnosis and Treatment of Peripheral Artery Diseases of the European Society of Cardiology (ESC). Eur Heart J 32(22):2851-2906

3. Rooke TW, Hirsch AT, Misra S, Sidawy AN, Beckman JA, Findeiss LK, Golzarian J, Gornik HL, Halperin JL, Jaff MR, Moneta GL, Olin JW, Stanley JC, White CJ, White JV, Zierler RE (2012) American College of Cardiology Foundation; American Heart Association; Society for Cardiovascular Angiography and Interventions; Society of Interventional Radiology; Society for Vascular Medicine; Society for Vascular Surgery. 2011 ACCF/AHA focused update of the guideline for the management of patients with peripheral artery disease (updating the 2005 guideline): a report of the American College of Cardiology Foundation/American Heart Association Task Force on Practice Guidelines: developed in collaboration with the Society for Cardiovascular Angiography and Interventions, Society of Interventional Radiology, Society for Vascular Medicine, and 
Society for Vascular Surgery. Catheter Cardiovasc Interv 79(4):501-531

4. Kashyap VS, Pavkov ML, Bishop PD, Nassoiy SP, Eagleton MJ, Clair DG, Ouriel K (2008) Angiography underestimates peripheral atherosclerosis: lumenography revisited. J Endovasc Ther 15(1):117-125

5. Halwani DO, Anderson PG, Brott BC, Anayiotos AS, Lemons JE (2012) The role of vascular calcification in inducing fatigue and fracture of coronary stents. J Biomed Mater Res B Appl Biomater 100(1):292-304

6. Adlakha S, Sheikh M, Wu J, Burket MW, Pandya U, Colyer W, Eltahawy E, Cooper CJ (2010) Stent fracture in the coronary and peripheral arteries. J Interv Cardiol 23(4):411-419

7. Shammas NW, Lam R, Mustapha J, Ellichman J, Aggarwala G, Rivera E, Niazi K, Balar N (2012) Comparison of orbital atherectomy plus balloon angioplasty vs. balloon angioplasty alone in patients with critical limb ischemia: results of the CALCIUM 360 randomized pilot trial. J Endovasc Ther 19(4):480-488

8. Fitzgerald PJ, Ports TA, Yock PG (1992) Contribution of localized calcium deposits to dissection after angioplasty. Circulation 86(1):64-70

9. Cioppa A, Stabile E, Popusoi G, Salemme L, Cota L, Pucciarelli A, Ambrosini V, Sorropago G, Tesorio T, Agresta A, Biamino G, Rubino P (2012) Combined treatment of heavy calcified femoropopliteal lesions using directional atherectomy and a paclitaxel coated balloon: one-year single centre clinical results. Cardiovasc Revasc Med 13(4):219-223

10. Amighi J, Schillinger M, Dick P, Schlager O, Sabeti S, Mlekusch W, Haumer M, Mathies R, Heinzle G, Schuster A, Loewe C, Koppensteiner R, Lammer J, Minar E, Cejna M (2008) De novo superficial femoropopliteal artery lesions: peripheral cutting balloon angioplasty and restenosis rates-randomized controlled trial. Radiology 247(1):267-272

11. Banerjee S, Das TS, Abu-Fadel MS, Dippel EJ, Shammas NW, Tran DL, Zankar A, Varghese C, Kelly KC, Weideman RA, Little BB, Reilly RF, Addo T, Brilakis ES (2012) Pilot trial of cryoplasty or conventional balloon post-dilation of nitinol stents for revascularization of peripheral arterial segments: the COBRA trial. J Am Coll Cardiol 60(15):1352-1359

12. Scheinert D, Peeters P, Bosiers M, O'Sullivan G, Sultan S, Gershony G (2007) Results of the multicenter first-in-man study of a novel scoring balloon catheter for the treatment of infrapopliteal peripheral arterial disease. Catheter Cardiovasc Interv 70(7):1034-1039

13. Werk M, Langner S, Reinkensmeier B, Boettcher HF, Tepe G, Dietz U, Hosten N, Hamm B, Speck U, Ricke J (2008) Inhibition of restenosis in femoropopliteal arteries: paclitaxel-coated versus uncoated balloon: femoral paclitaxel randomized pilot trial. Circulation 118(13):1358-1365

14. Tepe G, Zeller T, Albrecht T, Heller S, Schwarzwalder U, Beregi JP, Claussen CD, Oldenburg A, Scheller B, Speck U (2008) Local delivery of paclitaxel to inhibit restenosis during angioplasty of the leg. N Engl J Med 358(7):689-699

15. Fanelli F, Cannavale A, Corona M, Lucatelli P, Wlderk A, Salvatori FM (2014) The "debellum"-lower limb multilevel treatment with drug eluting balloon-randomized trial: 1-year results. J Cardiovasc Surg (Torino) 55(2):207-216
16. Micari A, Cioppa AMD, Vadalà G, Castriota F, Liso A, Marchese A, Grattoni C, Pantaleo P, Cremonesi A, Rubino P, Biamino G (2012) Clinical evaluation of a paclitaxel-eluting balloon for treatment of femoropopliteal arterial disease: 12-month results from a multicenter Italian registry. JACC Cardiovasc Interv 5(3):331-338

17. Stabile E, Virga V, Salemme L, Cioppa A, Ambrosini V, Sorropago G, Tesorio T, Cota L, Popusoi G, Pucciarelli A, Biamino G, Rubino P (2012) Drug-eluting balloon for treatment of superficial femoral artery in-stent restenosis. J Am Coll Cardiol 60(18):1739-1742

18. Werk M, Albrecht T, Meyer DR, Ahmed MN, Behne A, Dietz U, Eschenbach G, Hartmann H, Lange C, Schnorr B, Stiepani H, Zoccai GB, Hänninen EL (2012) Paclitaxel-coated balloons reduce restenosis after femoro-popliteal angioplasty: evidence from the randomized PACIFIER trial. Circ Cardiovasc Interv 5(6):831-840

19. Schmidt A, Piorkowski M, Werner M, Ulrich M, Bausback Y, Ick H, Schuster J, Botsios S, Kruse HJ, Varcoe RL, Scheinert D (2011) First experience with drug-eluting balloons in infrapopliteal arteries: restenosis rate and clinical outcome. J Am Coll Cardiol 58(11):1105-1109

20. Liistro F, Porto I, Angioli P, Grotti S, Ricci L, Ducci K, Falsini G, Ventoruzzo G, Turini F, Bellandi G, Bolognese L (2013) Drugeluting balloon in peripheral intervention for below the knee angioplasty evaluation (DEBATE-BTK): a randomized trial in diabetic patients with critical limb ischemia. Circulation 128(6):615-621

21. Scheller B, Speck U, Abramjuk C, Bernhardt U, Bohm M, Nickenig G (2004) Paclitaxel balloon coating, a novel method for prevention and therapy of restenosis. Circulation 110(7):810-814

22. Gertz ZM, Wilensky RL (2011) Local drug delivery for treatment of coronary and peripheral artery disease. Cardiovasc Ther 29(6):e54-e66

23. Scheller B (2011) Opportunities and limitations of drug-coated balloons in interventional therapies. Herz 36(3):232-239

24. Rocha-Singh KJ, Zeller T, Jaff MR (2014) Peripheral arterial calcification: prevalence, mechanism, detection, and clinical implications. Catheter Cardiovasc Interv. doi:10.1002/ccd.25387

25. Heijenbrok-Kal MH, Kock MC, Hunink MG (2007) Lower extremity arterial disease: multidetector CT angiography metaanalysis. Radiology 245(2):433-439

26. Raman R, Raman B, Napel S, Rubin GD (2008) Semiautomated quantification of the mass and distribution of vascular calcification with multidetector CT: method and evaluation. Radiology 247(1):241-250

27. Garcìa-Garcìa HM, Gogas BD, Serruys PW, Bruining N (2011) IVUS-based imaging modalities for tissue characterization: similarities and differences. Int J Cardiovasc Imaging 27(2):215-224

28. Mintz GS, Nissen SE, Anderson WD, Bailey SR, Erbel R, Fitzgerald PJ, Pinto FJ, Rosenfield K, Siegel RJ, Tuzcu EM, Yock PG (2001) American College of Cardiology Clinical Expert Consensus Document on Standards for Acquisition, Measurement and Reporting of Intravascular Ultrasound Studies (IVUS). A report of the American College of Cardiology Task Force on Clinical Expert Consensus Documents. J Am Coll Cardiol 37(5):1478-1492 